\title{
What are the Results of Resection of Forearm Soft Tissue Sarcoma?
}

Eiji Nakata ( $\square$ eijinakata8522@yahoo.co.jp)

Okayama University Hospital

\section{Tomohiro Fujiwara}

Okayama University Hospital

\section{Toshiyuki Kunisada}

Okayama University Hospital

\section{Ryuichi Nakahara}

Okayama University Hospital

\section{Toshiyuki Watanabe}

Okayama University Hospital

\section{Toshifumi Ozaki}

Okayama University Hospital

\section{Research Article}

Keywords: soft tissue sarcomas, surgery, Musculoskeletal Tumor Society rating scale, forearm, prognosis

Posted Date: November 1st, 2021

DOI: https://doi.org/10.21203/rs.3.rs-1028963/v1

License: (c) (i) This work is licensed under a Creative Commons Attribution 4.0 International License.

Read Full License 


\section{Abstract}

Only few reports have assessed the characteristics and oncological and functional outcomes of forearm soft tissue sarcomas (STS). Then, we aimed to investigate the clinical features and survival-related factors for forearm STS who underwent surgical excision at our institution. There were 38 patients. Fourteen patients (41\%) were referred to our institution after an unplanned excision and tumor size and grade were significantly associated with the receipt of it. The postoperative median Musculoskeletal Tumor Society rating scale (MSTS) score was 28 . Bone resection or major nerve palsy was the only factor influencing the postoperative MSTS score $(P<0.001)$. There was no significant difference in MSTS scores according to the reconstruction procedures (the use of flap or tendon reconstruction). The 5-year local recurrence-free survival (LRFS) rate was $86 \%$. Univariate analysis revealed that the histological diagnosis of myxofibrosarcoma was the only factor that influenced LRFS $(P=0.047)$. The 5 -year metastasis-free survival rate was $77 \%$. The 5 -year overall survival (OS) rate was $94 \%$. Age was the only factor that influenced OS $(P=0.01)$. In conclusion, reconstruction of the skin and tendon can compensate for function. Careful follow-up is important, especially in patients with myxofibrosarcoma, due to its likelihood of local recurrence.

\section{Introduction}

Soft tissue sarcomas (STS) of the forearm are rare, accounting for only $3-7 \%$ of all sarcomas ${ }^{1-6}$. Several reports have shown the functional and oncological results for the upper or distal upper $\operatorname{arm}^{7-10}$. However, limited reports have described the outcomes of STS arising in the forearm ${ }^{11}$. Some authors have reported a high percentage of unplanned excision without preoperative suspicion of malignancy in as much as $25-45 \%$ of patients with forearm STS ${ }^{9,11}$. The association between oncological outcomes and unplanned excision is controversial in STS. Some authors reported higher rates of local recurrence and worse local recurrence-free survival (LRFS) in patients with unplanned excision than in those who underwent planned excision; however, others reported no association between them ${ }^{12-15}$. Furthermore, the characteristics and factors associated with the receipt of unplanned excision have not been fully investigated in patients with forearm STS.

The goal of forearm STS treatment is to perform limb-sparing surgery with wide resection margins, while preserving the function of the distal upper extremity 4,5 . However, this becomes challenging because of the complex anatomy and limited tissue volume of the forearm. To obtain optimal oncological and functional outcomes, surgical excision with wide margins followed by reconstruction of the skin, tendons, nerves, vessels, bones, and joints should be performed. When tumors are locally aggressive or close to the major nerve and vessels, neoadjuvant treatments (chemotherapy, radiotherapy [RT], or a combination of both) are beneficial in some cases to avoid amputation.

To the best of our knowledge, only two reports have assessed the functional outcomes of forearm STS with a median score of 29.5 on the Musculoskeletal Tumor Society (MSTS) rating scale and 100 in the Toronto Extremity Salvage Score (TESS) ${ }^{9,11}$. However, no study has investigated the factors associated 
with functional outcomes in forearm STS. The oncological outcome of forearm STS is generally poor, with local recurrence occurring in 7-38\% of patients and distant metastases in $13-24 \%$ of patients ${ }^{7,9,11}$. However, the factors associated with these oncological outcomes have not been fully investigated.

Therefore, we analyzed the functional and oncological outcomes of forearm STS. For patients with forearm STS, we specifically asked the following: (1) What are the characteristics of unplanned versus planned excision? (2) Which factors, including the type of reconstruction, affect the functional outcomes? (3) What are the rates of LRFS, metastasis-free survival (MFS), and overall survival (OS) and the factors associated with these oncological outcomes?

\section{Results}

\section{Characteristics of unplanned excision in forearm STS}

Fourteen patients $(41 \%)$ were referred to our institution following an unplanned excision. Unplanned excision was performed by orthopedists in 10 patients, general surgeons in 3 patients, and plastic surgeons in 1 patient. MRI was performed preoperatively in seven patients $(50 \%)$ and ultrasonography of the tumor in one patient; no radiographic imaging was conducted in six patients. The median size of tumors in the unplanned excision group was significantly smaller than that of the planned excision group (3.2 $\mathrm{cm}$ [range, 1.1-9.5] and $6.1 \mathrm{~cm}$ [range, 2-13.8], respectively $[P<0.05])$. For four patients, those with tumors $<2 \mathrm{~cm}$ had received unplanned excision, whereas tumors $>10 \mathrm{~cm}$ were not excised. Subsequently, tumor size, grade, and stage were factors associated with the receipt of unplanned excision (Table 1). Tumors $<2 \mathrm{~cm}$ were seen in 4/14 (29\%) patients with unplanned excision, but none in those with planned excision $(P=0.02)$. A low Fédération Nationale des Centres de Lutte contre le Cancer (FNCLCC) grade (grade 1/2) was observed in 13/14 (93\%) patients with unplanned excision and 11/20 (55\%) with planned excision $(P=0.02)$. An early American Joint Committee on Cancer (AJCC) stage (stage IA/IB/II) was observed in $12 / 14(86 \%)$ patients with unplanned excision and in $8 / 20(40 \%)$ patients with planned excision $(P=0.01)$. The superficial STS were more likely to be excised inadvertently; the rates of unplanned excision in superficial and deep tumors were $12 / 23(52 \%)$ and $2 / 11(18 \%)$, respectively $(P=$ $0.08)$.

\section{Functional outcomes following resection of forearm STS}

The median MSTS score for all patients was 28 (range, 18-30). Bone resection or major nerve palsy was the only factor influencing the postoperative MSTS score; the median MSTS scores in patients with or without bone resection or major nerve disturbance were 24 (range, 18-25) and 29 (range, 18-30), respectively, which was significant $(P<0.001)$. There was no significant difference in MSTS scores according to the receipt of unplanned excision and reconstruction procedures (the use of flap or tendon reconstruction) (Table 2).

\section{LRFS, MFS, and OS in forearm STS}


Among the 34 patients, six (18\%) had a local recurrence, with a median recurrence period of 27 months (range, 3-91 months). Three patients underwent re-excision of the recurrent lesion; one patient remained disease-free even until the last follow-up. In one patient, local recurrence developed thrice, but the patient was disease-free at the last follow-up after repeated re-excision of these recurrences. One patient had local recurrence and intramuscular metastasis in the upper arm of the tumor-affected side and received disarticulation of the shoulder. One patient had local recurrence four times and subsequently underwent upper arm amputation. The 3 - and 5-year LRFS rates were $90 \%$ and $86 \%$, respectively (Fig. $1 \mathrm{~A})$. The histological diagnosis of myxofibrosarcoma was the only factor that influenced LRFS $(P=0.047)$ (Table 3). Among ten patients with myxofibrosarcoma, four experienced local recurrence, with the 3-and 5-year LRFS rates being $63 \%$ and $42 \%$, respectively (Fig. 1B). All patients with myxofibrosarcoma achieved R0 margins; furthermore, no patient received adjuvant RT. On the contrary, 2/22 patients with other tumors, 1 undifferentiated pleomorphic sarcoma (UPS) and 1 extraskeletal myxoid chondrosarcoma (EMC), showed local recurrence; moreover, the 3 - and 5-year LRFS rates were $100 \%$ and $85 \%$, respectively (Fig. 1B). Although the rate of local recurrence was higher in patients with R1 margins (67\%) than in those with R0 margins (13\%), this difference was not statistically significant $(P=0.07)$. None of the seven patients treated with RT experienced local recurrence, whereas $6 / 19$ patients treated with surgery alone experienced local recurrences; furthermore, we did not observe a significant association between local recurrence and the use of RT $(P=0.51)$.

Three patients had distant metastases at presentation; the lungs were involved in one of the patients, while the others had lymph node involvement. One patient with UPS had a single metastatic nodule in the lung upon initial presentation and, thus, underwent resection. Lung metastasis developed twice thereafter, but the patient became disease-free at the last follow-up, following re-resection of these metastatic nodules. One patient with alveolar rhabdomyosarcoma had a single lymph node metastasis and underwent both chemotherapy and RT; the patient was disease-free by the last follow-up. One patient with epithelioid sarcoma had a single lymph node metastasis and subsequently underwent chemotherapy and resection. Three months after the patient's surgery, multiple lung metastases developed, and the patient died a week later. Eight patients had distant metastases during the follow-up period, including six diagnosed with lung metastasis and two with lymph node metastasis. In three patients with two metastatic lung nodules, these lesions were resected; two patients remained diseasefree at the last follow-up, while one patient died due to multiple metastases. Three patients had multiple lung metastases 5 months, 5 months, and 13 years after surery, respectively, and subsequently died of the disease. Two patients had a single lymph node metastasis 16 and 17 months after surgery, respectively. These lesions were resected, and the patients remained disease-free at the last follow-up. The 3- and 5-year MFS rates were $77 \%$ and $77 \%$, respectively (Fig. 2). No factors were associated with MFS (Table 4).

Eight patients were dead by the last follow-up. The 3- and 5-year OS rates were $85 \%$ and $78 \%$, respectively (Fig. 3A). Age was the only factor that influenced OS $(P=0.01)$ (Table 5). Among 16 patients aged $\geq 65$ years, seven died; the 3 - and 5 -year OS rates were $75 \%$ and $60 \%$, respectively (Fig. 3B). Furthermore, one of 18 patients $<65$ years died. Thus, the 3 - and 5 -year OS rates were $94 \%$ and $94 \%$, respectively (Fig. 3B). 
We further investigated the characteristics of patients $<65$ years compared to those $\geq 65$ years in terms of tumor size, AJCC stage, FNCLCC grade, and treatment (chemotherapy and RT) using the Fisher's exact test (Supplementary Table S1). The older group had tumors of a higher grade (grade 3 ) than those in the younger group $(2 / 16$ [13\%] and $8 / 16[50 \%]$, respectively; $P=0.02)$. Fewer patients in the older group had received systemic chemotherapy than those in the younger group $(7 / 11$ [63\%] and 1/15 [7\%], respectively; $P=0.04)$.

\section{Discussion}

STS of the forearm is rare and characterized by a high likelihood of unplanned excision, high rate of local recurrence $(7-38 \%)$, and distant metastases $(13-24 \%)^{9,11}$. However, patient characteristics, factors associated with the receipt of unplanned versus planned excision, functional outcomes, and oncological outcomes have not been fully investigated. We found that $41 \%$ of forearm STS were referred to our institution following an unplanned excision, which was characterized by a tumor size $<2 \mathrm{~cm}$. The median MSTS score was 28 , which was influenced only by bone resection or major nerve palsy but not by reconstructive procedures (the use of flap and tendon reconstruction). We found that the 5-year LRFS, MFS, and OS rates were $86 \%, 77 \%$, and $78 \%$, respectively. The histological diagnosis of myxofibrosarcoma was the only factor that influenced LRFS, while age $\geq 65$ years was associated with an increased risk of OS.

Some authors reported a high percentage of unplanned excision without preoperative suspicion of malignancy in $25-45 \%{ }^{9}, 11$. In line with these reports, $41 \%$ of our patients were referred for an unplanned excision to manage their forearm STS. Furthermore, most unplanned excisions of forearm STS were probably performed without suspicion of malignancy, as only half of them received preoperative MRI. According to Baroudi et al., the characteristics of tumors with unplanned or planned excision differ in size ( $<4 \mathrm{~cm}$ in $81 \%$ and $23 \%$, respectively) and depth (superficial in $57 \%$ and $23 \%$, respectively) in patients with forearm STS ${ }^{11}$. We found that tumor size $\geq 2 \mathrm{~cm}$ was the only factor associated with the receipt of unplanned excision $(P=0.02)$. All patients with tumors $<2 \mathrm{~cm}$ underwent unplanned excision, whereas $10 / 30$ patients with tumors $\geq 2 \mathrm{~cm}$ underwent unplanned excision. The effect of unplanned excision on survival remains controversial. Potter et al. reported that the 5-year LRFS was higher in the unplanned excision group $(63.7 \%)$ than in the planned excision group $(89.7 \%)(P<0.0001)$ for patients with extremity and trunk STS ${ }^{13}$. Baroudi et al. reported that there was no difference in LRFS, MFS, and OS in patients with unplanned excision and planned excision for patients with forearm sarcoma ${ }^{11}$. We also found no difference in LRFS, MFS, and OS between patients with unplanned excision and planned excision. This can be explained by the tumors with unplanned excision being less aggressive (lower grade and earlier stage than tumors with planned excision), which could compensate the inadvertent treatment. Although the prognostic significance of unplanned excision should be further investigated, one should be careful in treating soft-tissue masses through conducting a comprehensive history taking and physical examination, requesting imaging tests, and referring these patients to a tertiary sarcoma center when they are suspected for a sarcoma. 
The goal of forearm STS treatment is to obtain wide resection margins of the primary tumor, while preserving upper limb function. However, it becomes challenging because of the complex anatomy and limited tissue volume of the forearm. Postoperative loss of tissues requires reconstruction of combined tissues, including the skin, tendons, nerves, vessels, bones, and joints. To achieve optimal oncological and functional outcomes, treatment strategies, including adjuvant treatments, should be defined by a multidisciplinary team including an oncological surgeon, hand surgeon, and plastic surgeon. These reconstructions greatly affect the postoperative functional outcome. Few studies have reported the functional outcomes of forearm STS. Muramatsu et al. reported median MSTS scores of 29.5 in eight patients who received microvascular reconstruction ( 5 free flaps) ${ }^{9}$. In the study by Bray et al., the functional outcomes were better in patients with forearm STS than in those with STS in the hand and wrist, with a mean TESS of 94 versus $88^{7}$. Certainly, it is difficult to accurately compare the functional outcomes of forearm STS with variable extents of excision, reconstruction, and functional scores (MSTS and TESS). However, these reports showed the possibility of good to excellent functional outcomes of forearm sarcoma, regardless of the receipt of adjuvant therapy or microvascular reconstruction. Similar to these reports, we found a median MSTS score of 28. Furthermore, we found that bone resection or major nerve palsy had significant effects on the MSTS score in forearm STS.

In the case of skin defects, which cannot be covered by split thickness skin graft (STSG), local pedicled or perforating flaps can be utilized ${ }^{16,17}$. However, they can increase the risk of cross-contamination. Thus, we usually use a free flap, commonly the anterolateral thigh (ALT). Other flaps such as latissimus dorsi (LD) flap, superficial circumflex iliac artery perforator (SCIP), abdominal, and inguinal are alternative candidates. Kang et al. reported that the flap reconstruction group had a lower MSTS score and higher wound complication rate but had better local control than those in the primary closure group in patients with STS of the upper extremity ${ }^{18}$. Others found that there was no significant difference in complication rate and functional outcomes between the pedicled and free flap groups in the upper extremity ${ }^{19,20}$. In this study, we first found equivalent limb function measured by MSTS with or without the use of a free flap in patients with forearm STS. Although partial necrosis was seen in two patients, they healed without any complication. Thus, the free flap is a safe and reliable procedure without impairing forearm function and minimal complications.

To the best of our knowledge, only one report has assessed the functional outcomes of forearm sarcoma with tendon reconstruction. Muramatsu et al. reported four cases with defects of the flexor or extensor forearm muscle after tumor resection that received functional neurovascular musculocutaneous flaps (free re-innervated transfer of gracilis or LD) to reconstruct finger flexors and extensors ${ }^{9}$. Reinnervation of the transferred muscle was obtained in all cases, and functional outcomes were evaluated as good to excellent, with a median MSTS score of $28(20-30)$. We usually reconstruct flexors, extensors, or both muscle groups (flexor digitorum profundus [FDP], extensor digitorum [ED], flexor pollicis longus [FPL], and extensor pollicis longus [EPL]) using tendon autografts or tendon transfer techniques. We reconstructed the tendon in eight patients who underwent resection of flexors and/or extensors (FDP, ED, FPL, and EPL). We found equivalent limb function measured by the MSTS in patients with or without tendon 
reconstruction, suggesting that the function of the forearm can be compensated by tendon reconstruction.

We found that bone resection or neurological disturbance (major nerve resection or paralysis) led to major loss of function, as median MSTS scores in patients with and without these factors were 24 (18$25)$ and 29 (18-30), respectively $(P<.001)$. In this study, three patients underwent ulnar resection, and among them, two patients lost their distal radioulnar joint (DRUJ). Although one patient received free vascularized fibular graft (FVFG) for the defect of the diaphysis of the ulna, this resulted in insufficient function. Preserving the major nerves is extremely important in the forearm, as sacrificing a major nerve leads to a major loss of function. Careful preoperative planning and adjuvant treatment may be necessary for preserving the major nerves. We performed epineural microsurgical dissection called in situ preparation (ISP) to avoid complete resection of major nerves when the tumor was located very close to them ${ }^{21}$. Invasion of nerve fibers by the tumor is rare, but the nerves can be preserved in most cases through this procedure. However, segmental resection cannot be avoided when the nerves are completely entrapped within the tumor. A nerve defect can be reconstructed using an interposition graft (sural nerve), distal nerve transfer, and vascularized nerve graft. However, the recovery of function is unpredictable; furthermore, its role in the reconstruction of major nerve defects has not yet been recognized ${ }^{5}$. In this study, postoperative neurological disturbance was observed in three patients without recovery, which led to severe hand dysfunction.

To the best of our knowledge, only one report has assessed survival and its associated risk factors in patients with forearm STS ${ }^{11}$. Baroudi et al. reported a local recurrence rate of $7 \%$ and a 5 -year LRFS rate of $94 \%{ }^{11}$. In this study, the local recurrence rate was $18 \%$, while the 5 -year LRFS rate was $86 \%$. The histological diagnosis of myxofibrosarcoma was the only factor that influenced LRFS. Myxofibrosarcoma has a locally infiltrative behavior and is associated with a high local recurrence rate of $24-44 \%^{22-24}$. Dadrass et al. reported that although positive resection margins were associated with local recurrence of myxofibrosarcoma, negative margins also resulted in a relatively high rate of local recurrence $(13 \%)$ compared to other soft tissue sarcoma subtypes ${ }^{22}$. In line with previous studies, a high rate of local recurrence was observed in this study; 4/10 myxofibrosarcoma patients experienced local recurrence, although all of them had achieved R0 margins. The 5-year LRFS rates of myxofibrosarcoma and others were $42 \%$ and $85 \%$, respectively $(P=0.047)$. With the small number of patients in this series, we could not show an association between local recurrence and resection margin or the use of RT. Although wide resection margins were presumed to result in good local control of forearm STS, no relationship between local recurrence and surgical margins was confirmed in this study. Baroudi et al. also reported no relationship between local recurrence and surgical margins in forearm STS ${ }^{11}$. Thus, the utility of surgical margins for local control of forearm STS should be investigated in a larger study. Locally aggressive STS, which makes limb-salvage difficult to achieve, may benefit from RT to avoid amputation in some cases. The role of RT in the treatment of extremity STS has been defined by two randomized clinical trials: RT reduced the risk of local recurrence in patients with high-grade tumors who had positive surgical margins $^{25,26}$. Its efficacy in local control does not differ from whether it is utilized before or after surgery, 
although there is a risk of postoperative complications with neoadjuvant $\mathrm{RT}^{25,26}$. The role of $\mathrm{RT}$ in the treatment of forearm STS has been previously investigated. Bray et al. reported local recurrence in 3/13 patients (two malignant fibrous tumors and one epithelioid sarcoma) who received neoadjuvant RT and tumor resection with negative margin for forearm STS ${ }^{7}$. In this study, none of the seven patients who received postoperative RT had local recurrence. Thus, the role of RT in the local control of forearm STS should be investigated further. Our treatment strategy for forearm STS is to perform R0 resection and use RT when the resection margins prove to be R1 or R2 because of the close proximity of the tumor to the major nerves or vessels.

Baroudi et al. reported a 5-year MFS rate of $74 \%$ and that the extra-compartment site was associated with a poor prognosis ${ }^{11}$. In this study, the 5 -year MFS rate was $77 \%$. Distant metastases were found in three patients upon initial presentation and in eight patients throughout the follow-up period. Among them, nine patients had lung metastases, with four undergoing radical pulmonary metastasectomy. Two patients remained disease-free by the last follow-up. Two patients who underwent resection of lymph node metastases continued to be disease-free during follow-up. Thus, radical metastasectomy is recommended in patients with forearm STS, when possible.

Baroud et al. reported a 5-year OS rate of $81 \%$ and described that large $(>4 \mathrm{~cm})$ and soft tissue reconstruction were associated with a poor prognosis ${ }^{11}$. In this study, the 5 -year OS rate was $77 \%$, while age ( $\geq 65$ years) was the only factor influencing OS. Among 16 patients aged $\geq 65$ years, seven died, and the 5 -year LRFS rate was $60 \%$. On the other hand, one of 18 patients aged $<65$ years died; thus, the 5 -year OS rate was $94 \%$. The negative impact of older age on the prognosis of patients with STS has been discussed in several reports ${ }^{27,28}$. Hoven-Gondrie et al. reported that patients $>65$ years were more likely to have an advanced-stage and high-grade STS ${ }^{28}$. They reported that the proportion of patients who received no treatment increased with age, which was significantly associated with relative 5 -year survival: $72.7 \%$ in younger patients and $43.8 \%$ in the elderly (cut-off: 85 years). In line with previous reports, we found high-grade sarcomas and less aggressive treatment in older patients than in younger patients, which would lead to lower OS in older patients than in younger patients. The efficacy of adjuvant chemotherapy with a combination of doxorubicin and ifosfamide was demonstrated in a metaanalysis ${ }^{29}$. Adjuvant chemotherapy has shown significant improvement in DFS and significantly prolonged OS, with the risk reduction improved to $11 \%$ for death and $12 \%$ for recurrence ${ }^{29}$. In this study, there was a trend toward superior OS in patients receiving chemotherapy, although we did not find a significant association between chemotherapy and OS. As other factors may influence this observation, larger studies are needed to confirm a possible association.

This study has several limitations. First, since forearm sarcoma is a rare malignancy, the sample size was small, with only 34 patients included; this limited our ability to identify factors associated with local recurrence and survival. Furthermore, some factors we explored were not necessarily associated with poor survival, and these factors may be important if conducted with a larger series. Second, wide resection was not performed in all patients, because some tumors close to major nerves and vessels were 
excised with R1 margins; this could have influenced survival rates. However, the sample size was likely insufficient to support this. Third, we investigated the function only by MSTS since we did not use other methods, such as TESS or disability of the arm, shoulder, and hand in all patients. We also did not investigate the grip power or other functions of the fingers. Finally, there may have been selection bias in our application of RT; we usually utilize RT in patients with surgical margins of R1 because of the close proximity of the tumor to major nerves or vessels. However, RT can influence the outcomes of local recurrence.

In conclusion, physicians should be careful in history taking and physical examination, requesting appropriate imaging studies, and referring them to a tertiary sarcoma center when they are suspected to have sarcomas. Resection with wide margins for the whole tumor is preferable; furthermore, RT should be utilized when marginal resection is performed because of the close proximity of the tumor to the major nerves or vessels. Soft tissue reconstruction using tendons can compensate for function, although bone resection and major nerve disturbances may aggravate the condition. The clinical behavior of forearm STS is relatively aggressive. Careful attention during follow-up is important, especially in patients with myxofibrosarcoma, to aid local control. Further studies based on a larger patient cohort would clarify the role of RT and surgical margins in this rare form of malignancy.

\section{Patients And Methods}

\section{Study population}

We retrospectively evaluated the medical records of patients with forearm STS who underwent surgery at our institution between October 1993 and March 2018. The forearm was defined as the area between the elbow and wrist creases. The inclusion criterion was a pathologically proven diagnosis of sarcoma. Patients excluded were those followed up for less than two years after surgery (three patients). During that time, 34 patients were treated for this diagnosis ( 21 men and 13 women; median range, 64 years [17 to 88]). Those living were followed up for a minimum of 24 months (median range, 70 months [4-296]) (Table 4).

\section{Imaging}

Computed tomography (CT) or 2-deoxy-2-(18F)fluoro-D-glucose positron emission tomography (FDGPET) combined with CT of the chest and abdomen were performed in all patients to determine the presence of distant metastasis upon initial presentation or at follow-up. We examined CT images of the chest, abdomen, and treated limb for all patients after surgery, while looking for distant metastasis every three to four months from the first to the third year of follow-up, twice a year until the fifth year, then once a year thereafter for high-grade sarcomas; we followed up the patients every six months in the first to the third year, then once a year thereafter for low-grade sarcomas. Magnetic resonance imaging (MRI) was used to evaluate the signal intensity, tumor size, and localization of the tumors.

\section{Diagnosis}


Histological diagnosis was established according to the WHO Classification of Tumors for all patients by expert pathologists ${ }^{30}$. The grade was determined using the FNCLCC grading system, which is based on tumor differentiation, mitosis count, and tumor necrosis ${ }^{31}$. Tumor size was defined as the maximal diameter on a cross-sectional MRI examination. Pathologic measurements were used to determine tumor size if pre-surgery cross-sectional imaging was not available in patients with unplanned excision.

Myxofibrosarcoma was most diagnosed in patients, accounting for ten patients, followed by UPS in eight patients, undifferentiated sarcoma, synovial sarcoma, and epithelioid sarcoma in three patients each, and one each for dermatofibrosarcoma protuberance, EMC, rhabdomyosarcoma, dedifferentiated liposarcoma, leiomyosarcoma, and clear cell sarcoma. Tumor size had a median range of $5.3 \mathrm{~cm}(1.1-$ 14), $<2 \mathrm{~cm}$ in four patients, $\geq 2 \mathrm{~cm}$ and $\leq 5 \mathrm{~cm}$ in 13 patients, $>5 \mathrm{~cm}$ and $\leq 10 \mathrm{~cm}$ in 13 patients, and $>10$ $\mathrm{cm}$ and $\leq 15 \mathrm{~cm}$ in four patients. Histopathology according to the FNCLCC grading system showed that the tumor was grade 1 in 7 patients, grade 2 in 17, and grade 3 in 10. AJCC stages were IA in three patients, IB in four, II in 13, IIIA in seven, IIIB in four, and IV in three. Tumors were superficial in 23 patients (68\%) and deep in 11 patients (32\%). The main lesion was located on the dorsal side of the forearm in 13 patients (38\%) and the palmar side in 21 patients (62\%). The right side was affected in 18 patients $(53 \%)$, and the left side was affected in 16 patients (47\%), with 19 patients (56\%) having their dominant hand involved (18 right-sided, one left-sided).

\section{Surgical treatment}

All patients initially underwent limb-sparing surgery. In patients with tumors close to major nerves and vessels, we utilized "ISP" to obtain wide margins ${ }^{21}$. The surgical procedure was as follows: The tumor and its surrounding tissues were excised en bloc to maintain the continuity of vessels and nerves. The mass was then isolated from the surgical bed using a vinyl sheet to prevent contamination. Thereafter, the vascular (nerve) sheath was split to release the vessels from the tumor. If the surface of the tumor was not exposed during this procedure, then the resected mass was removed from the operative field. If the vessels (nerve) completely adhered to the tumor, they were sacrificed. This method was performed on 12 patients.

Surgical excision margins were estimated based on the AJCC residual tumor classification ( $R$ classification) ${ }^{32}$. These surgical margins classified an R0 margin as free of malignancy, R1 margin as microscopic tumor cells present at the inked border of the specimen, and R2 as grossly positive margins. The surgical margins were estimated as R0 in 31 patients and R1 in 3 patients.

\section{Reconstructions}

Although soft tissue coverage was necessary in 25 patients, primary closure was achieved in all patients: 18 free flaps (ALT flap in 15 patients, LD flap in 2, SCIP flap in 1), rotation flap from the upper arm in 1 , and STSG in 6. We usually reconstruct flexor and extensor tendon defects of the fingers (FDP, ED), thumb (FPL, and EPL), and wrist when they are severely sacrificed. In cases of tendon defects without muscle belly defects, reconstruction is performed with standard tendon autografts (palmaris longus and fascia 
latae). If the muscle bellies must be sacrificed, then we utilize standard tendon transfer techniques for these cases, including radial nerve palsy, median nerve palsy, or brachial plexus injuries ${ }^{33,34}$. We performed tendon reconstruction in eight patients (finger in four patients, thumb in one patient, and both in three patients) (Supplementary Table S2). Six patients underwent primary surgery, and two patients underwent secondary surgery ( 6 months and 11 months after surgery for recurrence). ED was reconstructed in four patients by transfer to FCR (two patients), extensor carpi radialis longus (one patient), and extensor indicis proprius (one patient). FDP was reconstructed in three patients: autograft of the iliotibial band in two patients and transfer to the flexor digitorum superficialis in one patient. FPL was reconstructed in one patient by transfer to the flexor carpi ulnaris. EPL was reconstructed in three patients by transfer to the palmaris longus. Three patients underwent ulnar resection to achieve adequate margins. Two patients underwent bone resection upon their initial surgery, while the other had it upon recurrence. One patient underwent reconstruction with FVFG for defects of the diaphysis of the ulna; another patient underwent reconstruction of the DRUJ to resect the distal ulna using the Sauve-Kapandji method. One patient underwent resection of the diaphysis of the distal ulna, without reconstruction upon recurrence. In nine patients, the radial and ulnar arteries were sacrificed without vascular reconstruction in five and four patients, respectively, as estimated by the preoperative enhanced CT and Allen's test. In one patient, the ulnar nerve was sacrificed due to tumor adherence, without reconstruction.

\section{Postoperative complications}

Postoperative complications occurred in six patients (28\%). These complications were associated with tendon resection in one patient, flap reconstruction in two patients, and neurological disturbance in three patients. One patient developed finger contractures after tendon resection. Partial necrosis of the flap occurred in two patients; one patient with a pedicled flap healed with conservative treatment, while one with a free flap healed by debridement. Postoperative nerve palsy occurred in three patients. Ulnar nerve palsy was seen in two patients due to the sacrifice of the ulnar nerve in one and surgical procedure in the other; both patients did not recover. Posterior interosseous nerve palsy was observed in one patient, which did not improve with secondary neurolysis. Nonetheless, we found no tendon ruptures after tendon reconstruction and no complications associated with the ISP. Blood supply was unaffected in any patients without vascular reconstruction whose vessels were sacrificed.

\section{Adjuvant and neoadjuvant therapy}

After margins were obtained during surgery, we typically used conventional RT because of the close proximity of the tumor to the major nerves or vessels. RT was administered to seven patients $(21 \%)$ : one patient preoperatively and six patients postoperatively. The radiation absorbed dose was 40-66 Gy with a boost. Chemotherapy was administered to eight patients (24\%): one patient as a neoadjuvant, two patients as adjuvant, and five patients used a combination of both. The regimen comprised vincristine, doxorubicin, cyclophosphamide, ifosfamide, and etoposide for alveolar rabdomyosarcoma and doxorubicin and ifosfamide for the others. 


\section{Assessment of study outcomes}

To investigate the clinical characteristics of the unplanned and planned excision groups, we utilized the following variables: age, sex, FNCLCC grade, AJCC stage, tumor size, depth, and location of the tumor. Functional outcomes of the forearm after surgery were investigated using the MSTS rating scale ${ }^{35}$. We utilized the MSTS score at the last follow-up. In amputation cases, the best MSTS score was investigated during the follow-up period.

We determined the association of the following variables with the MSTS score: age, unplanned excision, resection of bone/major nerve palsy, the use of free flap, tendon reconstruction, receipt of chemotherapy, and RT.

We analyzed LRFS, MFS, and OS. LRFS was calculated from the date of surgery to the date of local recurrence or to the date of the last follow-up. Those who died without recurrence or metastases were censored on the date of their death. MFS was calculated from the date of diagnosis to the date of metastasis diagnosis or the last follow-up. OS was calculated from the date of diagnosis to the date of death or the last follow-up visit. Survival rates were estimated using the Kaplan-Meier method. We determined the association of the following variables in terms of survival: age, sex, histology, tumor size, FNCLCC grade, surgical margin, unplanned excision, metastasis upon initial presentation, receipt of chemotherapy, and RT.

The Mann-Whitney $U$ test was used to analyze continuous parameters, while Fisher's exact test was used for categorical parameters. For all analyses, associations were considered significant at a $P<0.05$, and we used the Bell Curve for Excel (Social Survey Research Information Co., Ltd., Tokyo, Japan).

\section{Declarations}

\section{Acknowledgements:}

We would like to thank Editage (www.editage.com) for English language editing.

\section{Authors' contributions}

Eiji Nakata, Toshiyuki Kunisada, and Toshifumi Ozaki organized the study. Eiji Nakata, Tomohiro Fujiwara, Toshiyuki Watanabe, and Toshiyuki Kunisada treated the patients. Eiji Nakata and Ryuichi Nakahara collected and analyzed data.

\section{Funding}

All authors have no funding in this study.

\section{Conflicts of interest}


Each author certifies that he or she has no commercial associations (eg, consultancies, stock ownership, equity interest, patent/licensing arrangements, etc) that might pose a conflict of interest in connection with the submitted article.

\section{Consent to participate}

Written informed consent was obtained from each participant included in this study.

\section{Consent for publication}

All co-authors agreed to the final version of the manuscript.

\section{Ethical approval statement}

This retrospective chart review study involving human participants was in accordance with the ethical standards of the institutional and national research committee and with the 1964 Helsinki Declaration and its later amendments or comparable ethical standards. The Human Investigation Committee (IRB) of Okayama University Hospital approved this study (approval number K2104-020)

\section{Data availability}

The datasets used and analyzed during the current study are available from the corresponding author on reasonable request.

\section{References}

1. Collin C, Hajdu SI, Godbold J, Friedrich C, Brennan MF. Localized operable soft tissue sarcoma of the upper extremity. Presentation, management, and factors affecting local recurrence in 108 patients. Ann Surg. 205, 331-339. https://10.1097/00000658-198704000-00001 (1987).

2. Duran-Moreno J, Kontogeorgakos V, Koumarianou A. Soft tissue sarcomas of the upper extremities: Maximizing treatment opportunities and outcomes. Oncol Lett. 18, 21792191. https://10.3892/ol.2019.10575 (2019).

3. Gustafson P and Arner M. Soft tissue sarcoma of the upper extremity: Descriptive data and outcome in a population-based series of 108 adult patients. J Hand Surg Am. 24, 668-674. https:// 10.1053/jhsu.1999.0668 (1999).

4. Koulaxouzidis G, Simunovic F, Bannasch H. Soft Tissue Sarcomas of the Arm - Oncosurgical and Reconstructive Principles within a Multimodal, Interdisciplinary Setting. Front Surg. 23, 12. https://10.3389/fsurg.2016.00012 (2016).

5. Lazerges C. Soft tissue sarcomas of the forearm, wrist and hand. Hand Surg Rehabil. 36, 233243. https://10.1016/j.hansur.2016.12.010 (2017).

6. Muramatsu K., et al. Musculoskeletal sarcomas in the forearm and hand: standard treatment and microsurgical reconstruction for limb salvage. Anticancer Res. 33, 4175-4182. (2013). 
7. Bray PW, Bell RS, Bowen CV, Davis A, O'Sullivan B. Limb salvage surgery and adjuvant radiotherapy for soft tissue sarcomas of the forearm and hand. $J$ Hand Surg Am. 22, 495503. https://10.1016/S0363-5023(97)80019-6 (1997).

8. Mundinger GS, Prucz RB, Frassica FJ, Deune EG. Concomitant upper extremity soft tissue sarcoma limb-sparing resection and functional reconstruction: assessment of outcomes and costs of surgery. Hand. 9, 196-204. https://10.1007/s11552-013-9567-9 (2014).

9. Muramatsu K, Ihara K, Doi K, Hashimoto T, Taguchi T. Sarcoma in the forearm and hand: clinical outcomes and microsurgical reconstruction for limb salvage. Ann Plast Surg. 62, 2833. https://10.1097/SAP.0b013e3181743a11 (2009).

10. Vetter M, Germann G, Bickert B, Sauerbier M. Current strategies for sarcoma reconstruction at the forearm and hand. J Reconstr Microsurg. 26, 455-460. https://10.1055/s-0030-1254229 (2010).

11. Baroudi MR, et al. Forearm soft tissue sarcoma: tumors characteristics and oncologic outcomes following limb salvage surgery. J Surg Oncol. 110, 676-681. https://10.1002/jso.23686 (2014).

12. Morattel $B$, et al. Oncological outcome, functional results and costs after unplanned excision of musculoskeletal soft tissue sarcoma. Eur J Surg Oncol. 46, 898904. https://10.1016/j.ejso.2020.01.025 (2020).

13. Potter BK, Adams SC, Pitcher JD Jr, Temple HT. Local recurrence of disease after unplanned excisions of high-grade soft tissue sarcomas. Clin Orthop Relat Res. 466, 30933100. https://10.1007/s11999-008-0529-4 (2008).

14. Pretell-Mazzini J, Barton MD Jr, Conway SA, Temple HT. Unplanned excision of soft-tissue sarcomas: current concepts for management and prognosis. J Bone Joint Surg Am. 977, 597603. https://10.2106/JBJS.N.00649 (2015).

15. Qureshi YA, et al. Unplanned excision of soft tissue sarcoma results in increased rates of local recurrence despite full further oncological treatment. Ann Surg Oncol. 19, 871877. https://10.1245/s10434-011-1876-z (2012).

16. Lee N, Roh S, Yang K, Kim J. Reconstruction of hand and forearm after sarcoma resection using anterolateral thigh free flap. J Plast Reconstr Aesthet Surg. 62, 584586. https://10.1016/j.bjps.2008.11.118 (2009).

17. Lucattelli $E$, et al. Reconstruction of upper limb soft-tissue defects after sarcoma resection with free flaps: A systematic review. J Plast Reconstr Aesthet Surg. 74, 755767. https://10.1016/j.bjps.2020.10.065 (2021).

18. Kang $S$, et al. Outcomes after flap reconstruction for extremity soft tissue sarcoma: a case-control study using propensity score analysis. Eur J Surg Oncol. 40, 11011108. https://10.1016/j.ejso.2014.05.004 (2014).

19. Payne $C E$, et al. Functional outcome following upper limb soft tissue sarcoma resection with flap reconstruction. $J$ Plast Reconstr Aesthet Surg. 66, 601-607. https://10.1016/j.bjps.2013.01.034 (2013). 
20. Slump J, et al. Flap choice does not affect complication rates or functional outcomes following extremity soft tissue sarcoma reconstruction . J Plast Reconstr Aesthet Surg. 71, 989996. https://10.1016/j.bjps.2018.04.002 (2018).

21. Matsumoto S, Kawaguchi N, Manabe J, Matsushita Y. "In situ preparation": new surgical procedure indicated for soft-tissue sarcoma of a lower limb in close proximity to major neurovascular structures. Int J Clin Oncol. 7, 51-56. https://10.1007/s101470200006 (2002).

22. Dadrass $F$, et al. A clinicopathologic examination of myxofibrosarcoma. Do surgical margins significantly affect local recurrence rates in this infiltrative sarcoma subtype? J Surg Oncol. 123, 489496. https://10.1002/jso.26277 (2021).

23. Lin CN, et al. Prognostic factors of myxofibrosarcomas: implications of margin status, tumor necrosis, and mitotic rate on survival. J Surg Oncol. 93, 294-303. https:// 10.1002/jso.20425 (2006).

24. Roland CL, Wang WL, Lazar AJ, Torres KE. Myxofibrosarcoma. Surg Oncol Clin N Am. 25, 775788. https://10.1016/j.soc.2016.05.008 (2016).

25. Pisters PW, et al. Long-term results of a prospective randomized trial of adjuvant brachytherapy in soft tissue sarcoma. J Clin Oncol. 14, 859-868. https://10.1200/JC0.1996.14.3.859 (1996).

26. Yang JC, et al. Randomized prospective study of the benefit of adjuvant radiation therapy in the treatment of soft tissue sarcomas of the extremity. J Clin Oncol. 16, 197203. https://10.1200/JC0.1998.16.1.197 (1998).

27. Biau DJ, et al. Adverse effect of older age on the recurrence of soft tissue sarcoma of the extremities and trunk. J Clin Oncol. 29, 4029-4035. https://10.1200/JC0.2010.34.0711 (2011).

28. Hoven-Gondrie ML, et al. Worse Survival in Elderly Patients with Extremity Soft-Tissue Sarcoma. Ann Surg Oncol. 23, 2577-2585. https://10.1245/s10434-016-5158-7 (2016).

29. Pervaiz N, et al. A systematic meta-analysis of randomized controlled trials for adjuvant chemotherapy for localized resectable soft tissue sarcoma. Cancer 113, 573581. https://10.1002/cncr.23592 (2008).

30. Fletcher CDM. WHO Classification of Tumours of soft tissue and bone. $5^{\text {th }}$ ed. Lion: IARC Press; (2020).

31. Guillou L, et al. Comparative study of the National Cancer Institute and French Federation of Cancer Centers Sarcoma Group grading systems in a population of 410 adult patients with soft tissue sarcoma. J Clin Oncol. 15, 350-362. https://10.1200/JC0.1997.15.1.350 (1997).

32. Wittekind $\mathrm{C}$, et al. A uniform residual tumor $(\mathrm{R})$ classification: integration of the $\mathrm{R}$ classification and the circumferential margin status. Cancer. 115, 3483-3488. https://10.1002/cncr.24320 (2009).

33. Jones NF, Machado GR. Tendon transfers for radial, median, and ulnar nerve injuries: current surgical techniques. Clin Plast Surg. 38, 621-642. https://10.1016/j.cps.2011.07.002 (2011).

34. Seiler JG 3rd, Desai MJ, Payne SH. Tendon transfers for radial, median, and ulnar nerve palsy. J Am Acad Orthop Surg. 21, 675-684. https://10.5435/JAAOS-21-11-675 (2013). 
35. Enneking WF, Dunham W, Gebhardt MC, Malawar M, Pritchard DJ. A system for the functional evaluation of reconstructive procedures after surgical treatment of tumors of the musculoskeletal system. Clin Orthop Relat Res. 286, 241-246. (1993).

\section{Tables}

Table 1. Associated factors of unplanned excision

\begin{tabular}{|c|c|c|c|c|}
\hline Variable & Category & Patients, number & & \\
\hline & & $\begin{array}{l}\text { Patients with } \\
\text { unplanned excision }\end{array}$ & $\begin{array}{l}\text { Patients without } \\
\text { unplanned excision }\end{array}$ & p-Value \\
\hline Age, years & $<65$ & 9 & 9 & 0.31 \\
\hline & ${ }^{3} 65$ & 5 & 11 & \\
\hline Sex & Male & 7 & 13 & 0.49 \\
\hline & Female & 7 & 7 & \\
\hline Tumor size & $<2 \mathrm{~cm}$ & 4 & 0 & 0.02 \\
\hline & ${ }^{3} 2 \mathrm{~cm}$ & 10 & 20 & \\
\hline
\end{tabular}

$\begin{array}{lllll}\text { FNCLCC grade } & \text { Grade } 1,2 & 13 & 11 & 0.02 \\ & \text { Grade } 3 & 1 & 9 & \end{array}$

\begin{tabular}{lllll}
\hline AJCC stage & IA, IB, II & 12 & 8 & 0.01 \\
\hline & IIIA, IIIB, IV & 2 & 12 & \\
\hline
\end{tabular}

\begin{tabular}{lllll}
\hline Depth & Superficial & 12 & 11 & 0.08 \\
\hline & Deep & 2 & 9 & \\
\hline Location & Palmor & 8 & 13 & 0.73 \\
\hline & Dorsal & 6 & 7 &
\end{tabular}


FNCLCC; Fédération Nationale des Centres de Lutte contre le Cancer AJCC; American Joint Committee on Cancer

Table 2. Risk factors of MSTS 


\begin{tabular}{llll} 
Variable & Category & Median & p-Value \\
\hline Age, years & $<65$ & $29(20-30)$ & 0.83 \\
\hline & ${ }^{3} 65$ & $27(18-30)$ &
\end{tabular}

\begin{tabular}{llll}
\hline Unplanned excision & Yes & $29(20-30)$ & 0.40 \\
\hline No & $28(18-30)$ & \\
\hline
\end{tabular}

\begin{tabular}{llll}
\hline Resection of bone/nerve palsy & Yes & $24(18-25)$ & 0.001 \\
\hline No & $29(24-30)$ \\
\hline
\end{tabular}

$\begin{array}{lllll}\text { Free flap } & \text { Yes } & 28(20-30) & 0.64 & \begin{array}{l}\text { Table 3. Risk factors of local } \\ \text { recurrence }\end{array} \\ \text { No } & 29(18-30) & \end{array}$

\begin{tabular}{llll}
\hline Tendon reconstruction & Yes & $29(22-30)$ & 0.80 \\
\hline & No & $28(18-30)$
\end{tabular}

\begin{tabular}{llll} 
Chemotherapy & Yes & $29(20-30)$ & 0.43 \\
& No & $28(18-30)$ & \\
\hline & & & \\
Radiotherapy & Yes & $29(20-30)$ & 0.86 \\
\hline No & $28(18-30)$ &
\end{tabular}




\begin{tabular}{|c|c|c|c|c|}
\hline \multirow[t]{3}{*}{ Variable } & \multirow[t]{3}{*}{ Category } & \multicolumn{3}{|c|}{ Patients, number } \\
\hline & & Patients with & $\begin{array}{l}\text { Patients } \\
\text { without }\end{array}$ & $\begin{array}{l}\mathrm{p}- \\
\text { Value }\end{array}$ \\
\hline & & $\begin{array}{l}\text { local } \\
\text { recurrence }\end{array}$ & $\begin{array}{l}\text { local } \\
\text { recurrence }\end{array}$ & \\
\hline \multirow[t]{2}{*}{ Age, years } & $<65$ & 4 & 14 & 0.66 \\
\hline & ${ }^{3} 65$ & 2 & 14 & \\
\hline \multirow[t]{2}{*}{ Sex } & Male & 3 & 17 & 0.67 \\
\hline & Female & 3 & 11 & \\
\hline \multirow[t]{2}{*}{ Histology } & Myxofibrosarcoma & 4 & 6 & 0.047 \\
\hline & Others & 2 & 22 & \\
\hline \multirow[t]{2}{*}{ Tumor size } & $<2 \mathrm{~cm}$ & 1 & 3 & 0.96 \\
\hline & ${ }^{3} 2 \mathrm{~cm}$ & 5 & 25 & \\
\hline \multirow[t]{2}{*}{ FNCLCC grade } & Grade 1 & 3 & 4 & 0.08 \\
\hline & Grade 2,3 & 3 & 24 & \\
\hline \multirow[t]{2}{*}{ Margin } & RO & 4 & 27 & 0.07 \\
\hline & $\mathrm{R} 1$ & 2 & 1 & \\
\hline \multirow[t]{2}{*}{ Unplanned excision } & Yes & 4 & 10 & 0.51 \\
\hline & No & 2 & 18 & \\
\hline \multirow{2}{*}{$\begin{array}{l}\text { Metastases upon initial } \\
\text { presentation }\end{array}$} & Yes & 2 & 1 & 0.30 \\
\hline & No & 4 & 27 & \\
\hline
\end{tabular}


Radiotherapy

Yes

No
0

6
0.31

21

FNCLCC; Fédération Nationale des Centres de Lutte contre le Cancer

Table 4. Risk factors of metastases free survival 


\begin{tabular}{|c|c|c|c|c|}
\hline \multirow[t]{2}{*}{ Variable } & \multirow[t]{2}{*}{ Category } & \multicolumn{3}{|c|}{ Patients, number } \\
\hline & & $\begin{array}{l}\text { Patients with } \\
\text { metastases }\end{array}$ & $\begin{array}{l}\text { Patients without } \\
\text { metastases }\end{array}$ & p-Value \\
\hline \multirow[t]{2}{*}{ Age, years } & $<65$ & 4 & 13 & 1.00 \\
\hline & ${ }^{3} 65$ & 4 & 10 & \\
\hline \multirow[t]{2}{*}{ Sex } & Male & 5 & 12 & 0.70 \\
\hline & Female & 3 & 11 & \\
\hline \multirow[t]{2}{*}{ Histology } & Myxofibrosarcoma & 2 & 8 & 0.69 \\
\hline & Others & 6 & 15 & \\
\hline \multirow[t]{2}{*}{ Tumor size } & $<2 \mathrm{~cm}$ & 0 & 4 & 0.55 \\
\hline & ${ }^{3} 2 \mathrm{~cm}$ & 8 & 19 & \\
\hline \multirow[t]{2}{*}{ FNCLCC grade } & Grade 1 & 2 & 5 & 1.00 \\
\hline & Grade 2,3 & 6 & 18 & \\
\hline \multirow[t]{2}{*}{ Margin } & RO & 7 & 21 & 1.00 \\
\hline & $\mathrm{R} 1$ & 1 & 2 & \\
\hline
\end{tabular}

$\begin{array}{cccccc}\begin{array}{c}\text { Unplanned excision } \\ \text { Yes }\end{array} & 3 & 11 & 0.70 & \begin{array}{l}\text { Risk } \\ \text { factors of } \\ \text { overall }\end{array} \\ & \text { No } & 5 & 12 & \text { survival }\end{array}$

\begin{tabular}{lllll}
\hline Chemotherapy & Yes & 1 & 5 & 1.00 \\
\hline & No & 7 & 18
\end{tabular}

$\begin{array}{llllll}\text { Radiotherapy } & \text { Yes } & 2 & 4 & 0.63 & \begin{array}{l}\text { Fédération } \\ \text { Nationale } \\ \text { No }\end{array} \\ & 6 & 19 & & \text { des Centres }\end{array}$


contre le Cancer

\section{Figures}
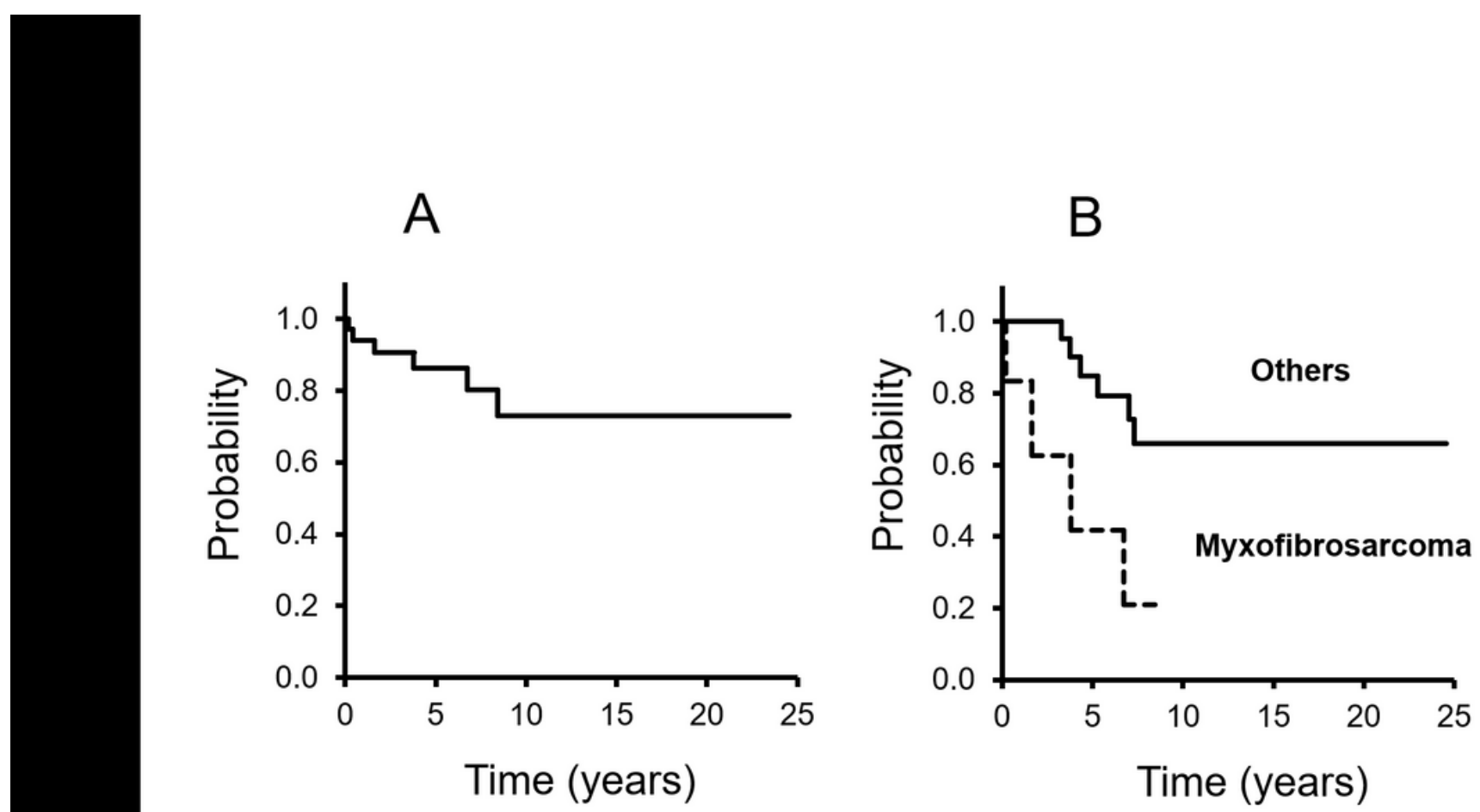

\section{Figure 1}

Kaplan-Meier curves for local recurrence-free survival (LRFS). (A) All patienst. The 3- and 5-year LRFS rates are $90 \%$ and $86 \%$, respectively.; (B) Patients with myxofibrosarcoma and others. The 5-year LRFS rates are $42 \%$ and $85 \%$ in patients with myxofibrosarcoma and others, respectively. 


\begin{tabular}{|c|c|c|c|c|}
\hline \multirow[t]{2}{*}{ Variable } & \multirow[t]{2}{*}{ Category } & \multicolumn{3}{|c|}{ Patients, number } \\
\hline & & $\begin{array}{l}\text { Patients with } \\
\text { death }\end{array}$ & $\begin{array}{l}\text { Patients without } \\
\text { death }\end{array}$ & $\begin{array}{l}\mathrm{p}- \\
\text { Value }\end{array}$ \\
\hline \multirow[t]{2}{*}{ Age, years } & $<65$ & 1 & 17 & 0.01 \\
\hline & ${ }^{3} 65$ & 7 & 9 & \\
\hline \multirow[t]{2}{*}{ Sex } & Male & 6 & 14 & 0.42 \\
\hline & Female & 2 & 12 & \\
\hline \multirow[t]{2}{*}{ Histology } & Myxofibrosarcoma & 1 & 9 & 0.39 \\
\hline & Others & 7 & 17 & \\
\hline \multirow[t]{2}{*}{ Tumor size } & $<2 \mathrm{~cm}$ & 0 & 4 & 0.55 \\
\hline & ${ }^{3} 2 \mathrm{~cm}$ & 8 & 22 & \\
\hline \multirow[t]{2}{*}{ FNCLCC grade } & Grade 1 & 1 & 6 & 1.00 \\
\hline & Grade 2,3 & 7 & 20 & \\
\hline \multirow[t]{2}{*}{ Margin } & Ro & 6 & 25 & 0.13 \\
\hline & R1 & 2 & 1 & \\
\hline \multirow[t]{2}{*}{ Unplanned excision } & Yes & 1 & 13 & 0.30 \\
\hline & No & 7 & 13 & \\
\hline \multirow{2}{*}{$\begin{array}{l}\text { Metastases upon initial } \\
\text { presentation }\end{array}$} & Yes & 2 & 1 & 0.13 \\
\hline & No & 6 & 25 & \\
\hline \multirow[t]{2}{*}{ Chemotherapy } & Yes & 1 & 7 & 0.20 \\
\hline & No & 7 & 19 & \\
\hline
\end{tabular}




\begin{tabular}{ccccc}
\hline Radiotherapy & Yes & 3 & 4 & 0.51 \\
\hline No & 5 & 22 &
\end{tabular}
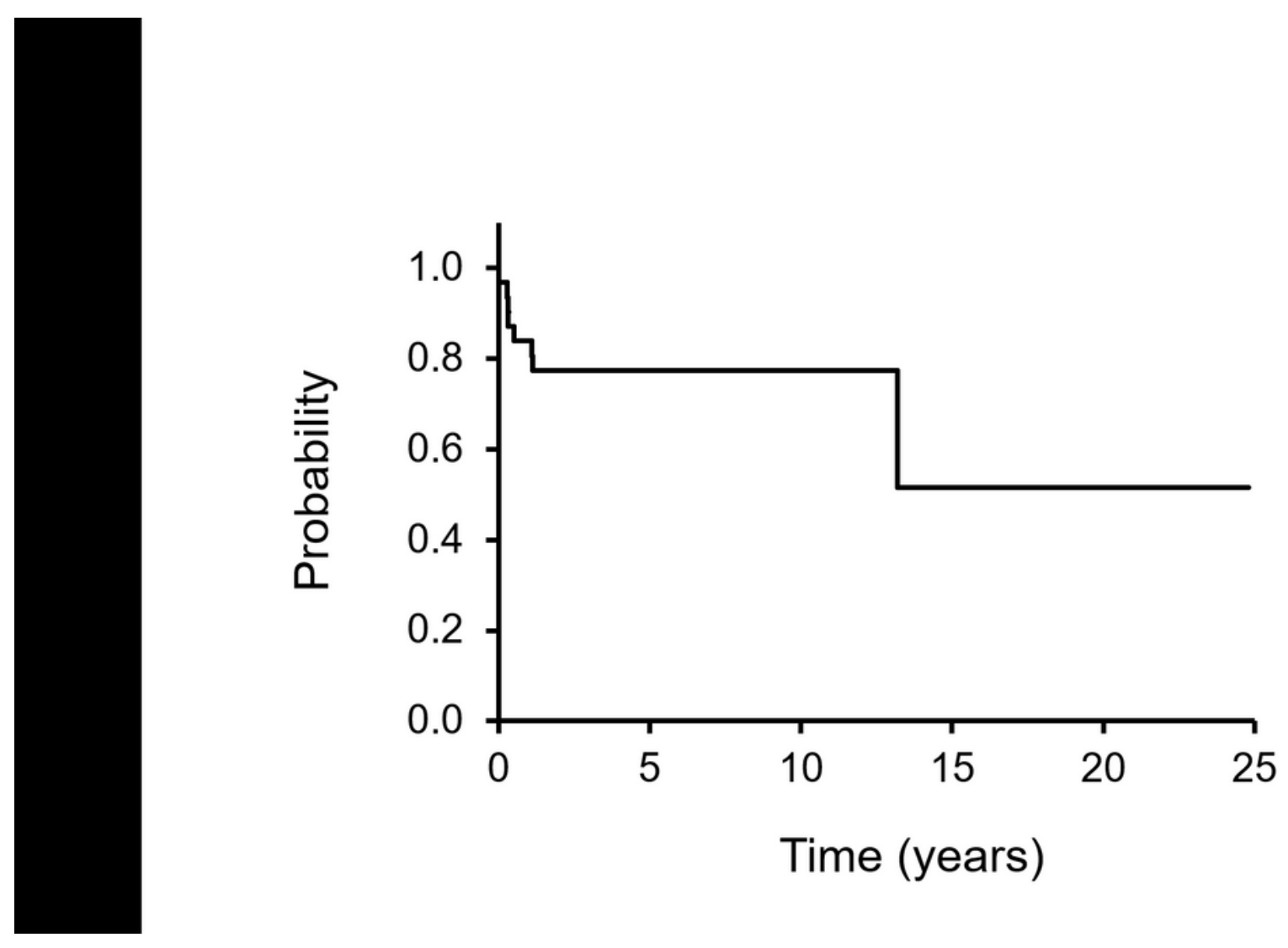

Figure 2

Kaplan-Meier curves for metastasis-free survival (MFS). The 3- and 5-year LRFS rates are $77 \%$ and $77 \%$, respectively. 

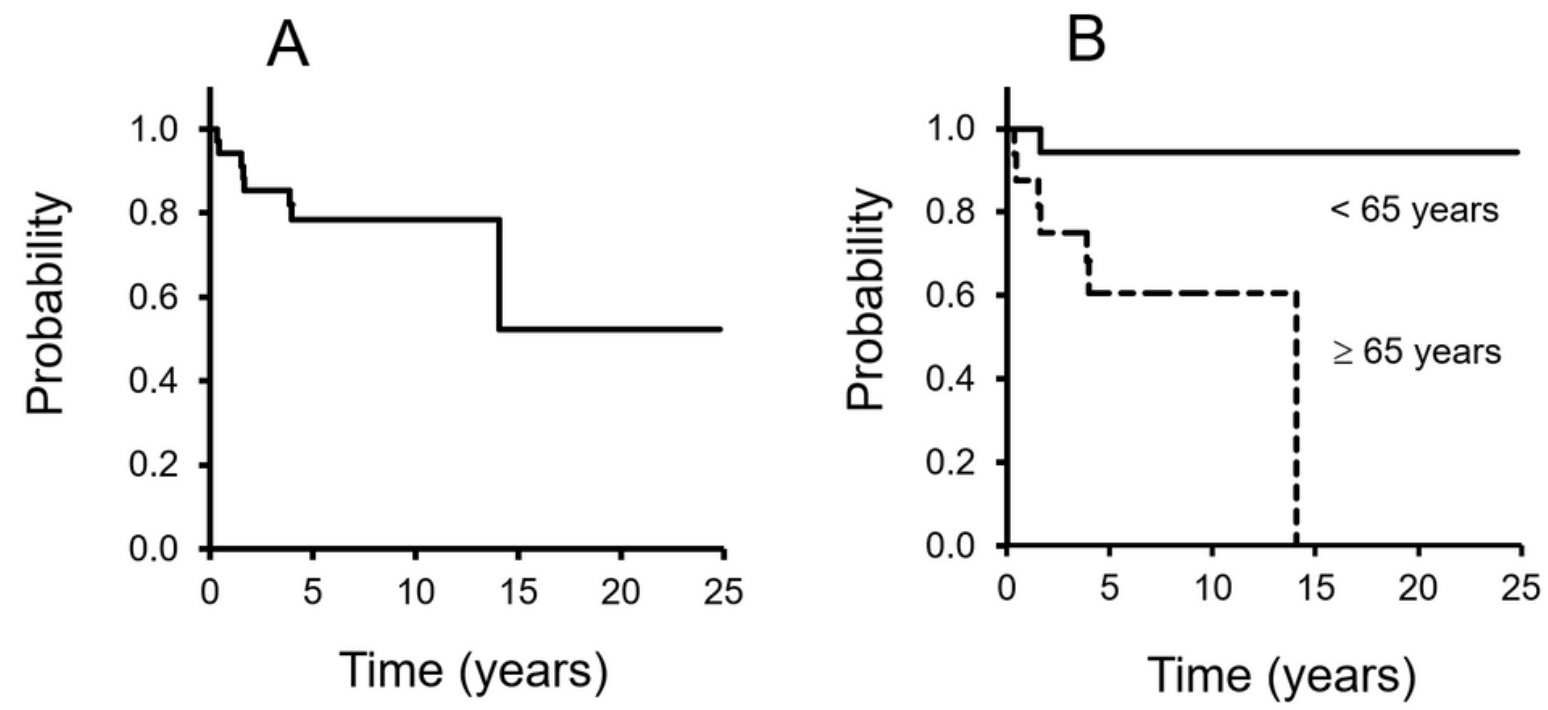

Figure 3

Kaplan-Meier curves for overall survival (OS). (A) All patients. The 3- and 5-year OS rates are $75 \%$ and $60 \%$, respectively.; (B) Patients with $\geq 65$ years and $\geq 65$ years. The 5 -year OS rates are $60 \%$ and $94 \%$ in patients $\geq 65$ years and $<65$ years, respectively.

\section{Supplementary Files}

This is a list of supplementary files associated with this preprint. Click to download.

- Supplementary.docx 